\title{
LA PROTECCIÓN FINANCIERA PARA LA GESTIÓN DEL RIESGO DE DESASTRES EN AMÉRICA LATINA
}

Cristian Camilo Fernández Lopera ${ }^{{ }^{*}}$

\section{RESUMEN}

Siendo América Latina una región afectada frecuentemente por desastres, se han gestado mecanismos de reducción de la vulnerabilidad como la Protección Financiera (PF). El propósito de esta investigación consistió en caracterizar la PF mediante la revisión de fuentes oficiales que dieron a conocer los principales actores involucrados, tipos de instrumentos, marcos regulatorios, avances y retos. Los principales actores son organismos multilaterales, empresas privadas y gobiernos nacionales. Los resultados muestran que la región no cuenta con un marco regulatorio común. Los retos se centran en la articulación sector público y privado, en la creación de marcos regulatorios y en el diseño de productos para grupos vulnerables.

\section{PALABRAS CLAVE}

América Latina, Gestión del riesgo de desastres, Transferencia del riesgo, Protección financiera, Reducción de la vulnerabilidad

\section{FINANCIAL PROTECTION FOR THE DISASTER RISK MANAGEMENT IN LATIN AMERICA}

\section{ABSTRACT}

As Latin America is a region frequently affected by disasters, vulnerability reduction mechanisms such as Financial Protection (FP) have been developed. The purpose of this research was to characterize the FP by reviewing official sources that made known the main actors involved, types of instruments, regulatory frameworks, advances and challenges. The main actors are multilateral organizations, private companies and national governments. The region does not have a common regulatory framework. The challenges focus on the articulation of the public and private sectors, on the creation of regulatory frameworks and the design of products for vulnerable groups.

\section{KEYWORDS}

Latin America, Disaster risk management, Risk transfer, Financial protection, Vulnerability reduction

\author{
1. Centro de Estudios \\ Sociales, Universidad de \\ Coimbra, Coimbra, Portugal. \\ *Autor de correspondencia: \\ camilofernandez@ces.uc.pt

\section{RECIBIDO} \\ 2 de marzo de 2020

\section{ACEPTADO} \\ 14 de abril de 2020

\section{PUBLICADO} \\ 1 de julio de 2020

\section{Formato cita} \\ Recomendada (APA): \\ Fernández Lopera , C.C. \\ (2020). La Protección \\ Financiera para la Gestión \\ del Riesgo de Desastres en \\ América Latina. Revista de \\ Estudios Latinoamericanos \\ sobre Reducción del Riesgo \\ de Desastres REDER, 4(2), \\ 22-35.
}

\section{(ब) $(\Phi \otimes$}

Todos los artículos publicados en REDER siguen una política de Acceso Abierto y se respaldan en una Licencia CreativeCommons Atribución-NoComercial 4.0 Internacional.

Revista de Estudios Latinoamericanos sobre Reducción del Riesgo de Desastres (REDER)

Diseño: Lupe Bezzina Tipografía: Hospital 


\section{INTRODUCCIÓN}

La ocurrencia de desastres se ha incrementado en los últimos 50 años debido principalmente a dos factores: aumento en el número de bienes 'expuestos' y aumento en la 'vulnerabilidad' de las personas, sus bienes y medios de subsistencia. De los 10.271 desastres ocurridos en el mundo en el periodo 1970-2010, el 16,9\% ocurrieron en América Latina y el Caribe (Bello, 2017), estos detonados por amenazas de origen climático que, según Bello (2017), para el mismo periodo han aumentado en un $309 \%$ en el Caribe y un $425 \%$ en Centroamérica (Figura 1). Centroamérica y Caribe son las subregiones más afectadas por desastres en América Latina. En especial la subregión Andina se caracteriza por escenarios multi-riesgo en la cual se han desarrollado de forma simultánea y/o concatenada, eventos naturales como sismos, tsunamis, movimientos en masa, inundaciones, heladas y sequías (CAN, 2009).

Según el Índice de Riesgo Climático Global 2019 (IRC) (National Academies of Sciences, Engineering, 2019), cinco (Puerto Rico, Honduras, Haití, Nicaragua y Dominica) de los 10 países más afectados entre 1998 y 2017 se encuentran en la región de América Latina y el Caribe, todos afectados por fenómenos derivados del régimen hidrológico (Figura 1) como ciclones tropicales, marejadas de tormenta, inundaciones y movimientos en masa. En el marco del cambio climático, se espera el aumento en la recurrencia e intensidad de los eventos climáticos extremos (National Academies of Sciences, Engineering, 2019), significando un aumento en la amenaza y por consiguiente de las condiciones de riesgo.

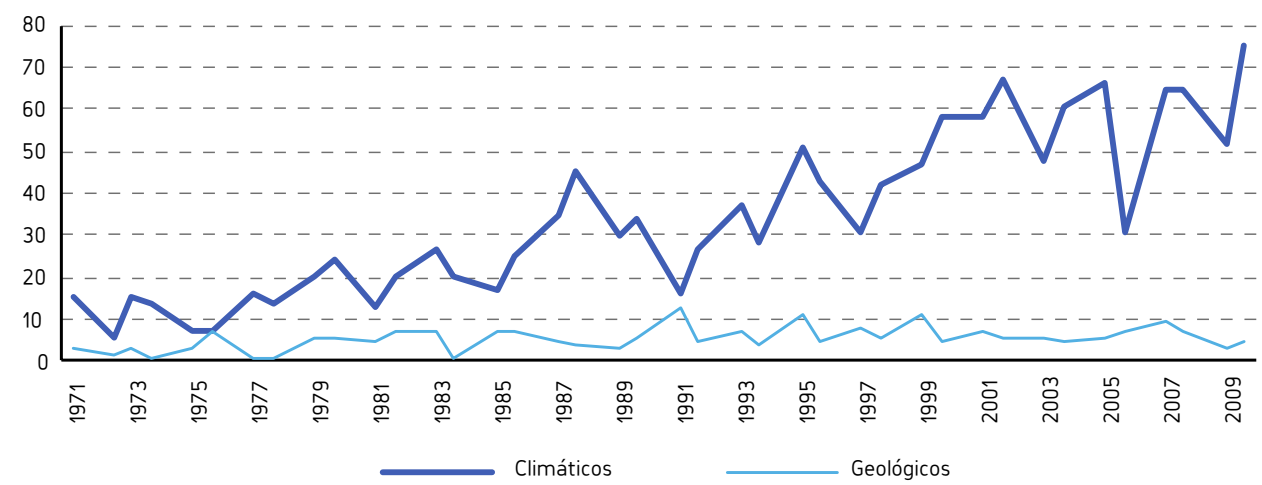

Figura 1. Número de desastres ocurridos entre los años 1970-2010 en América Latina y el Caribe Fuente: EMDAT, OFDA/CRED en Bello (2017)

La complejidad y el rápido crecimiento de las condiciones de riesgo en los países de América Latina exige enfoques multidisciplinarios que articulados, disminuyan las probabilidades de la ocurrencia de desastres a diferentes escalas territoriales y sectoriales. Para la implementación de enfoques alternativos, se acude a la Gestión del Riesgo de Desastres (GRD), definida como "El proceso sistemático de utilizar directrices administrativas, organizaciones, destrezas y capacidades operativas para ejecutar políticas y fortalecer las capacidades de afrontamiento, con el fin de reducir el impacto adverso de las amenazas naturales y la posibilidad de que ocurra un desastre" (UNDRR, 2009, p.19).

Con el objetivo de desarrollar políticas públicas de GRD integrales (evitando el enfoque centrado en la atención de las emergencias), en algunos países de América Latina se ha adoptado el enfoque de la GRD basado en procesos (Narváez et al., 2009), que consiste en una secuencia de insumos-productos expresada en procesos fundamentales como Conocimiento del Riesgo (CR); Reducción del Riesgo (RR), que contiene la planificación y la protección financiera y, el Manejo de los Desastres (MD). Este enfoque conceptual ha sido implementado en Perú (Congreso de la República del Perú, 2011), Colombia (Congreso de Colombia, 2012), Bolivia (Asamblea Legislativa Plurinacional de Bolivia, 2014) y Chile (Ministerio del Interior y Seguridad Pública, 2016).

En el marco del enfoque de la GRD por procesos, algunos modelos han vinculado al proceso de RR la Protección Financiera (PF), entendiendo esta como "Mecanismos o instrumentos financieros de retención intencional o transferencia del riesgo que se establecen en forma ex ante con el fin de acceder de manera ex post a recursos económicos oportunos para la atención de las emergencias y la recuperación" (UNGRD, 2017, p.29), Además, las acciones de PF pueden estar dirigidas a la implementación de acciones ex ante como a la prevención del riesgo (Miller \& Keipi, 2006). El Banco Mundial (BM) y el Fondo Mundial para la Reducción del Riesgo y la Recuperación (GFDRR por sus 
siglas en inglés) en su marco de referencia para la GRD, define a la PF como uno de los 5 pilares de la GRD' (Disaster Risk Financing and Insurance Program -DRFIP, 2020), hecho que indica la importancia de la PF para la RR.

La adquisición de instrumentos para la protección financiera se ha incrementado en países con ingresos medios bajos y bajos, situación que corresponde a los países en los que se ha presentado mayor número de muertes por desastres de origen natural y socionatural (Figura 2).

Generalmente en los países de bajos y medios ingresos, las acciones de PF con pagos rápidos mitigan los impactos económicos de los fenómenos, evitando que las emergencias se conviertan en desastres y que la recuperación se dé en periodos de tiempo más cortos (KfW Development Bank \& InsuResilience Global Partnership, 2019).

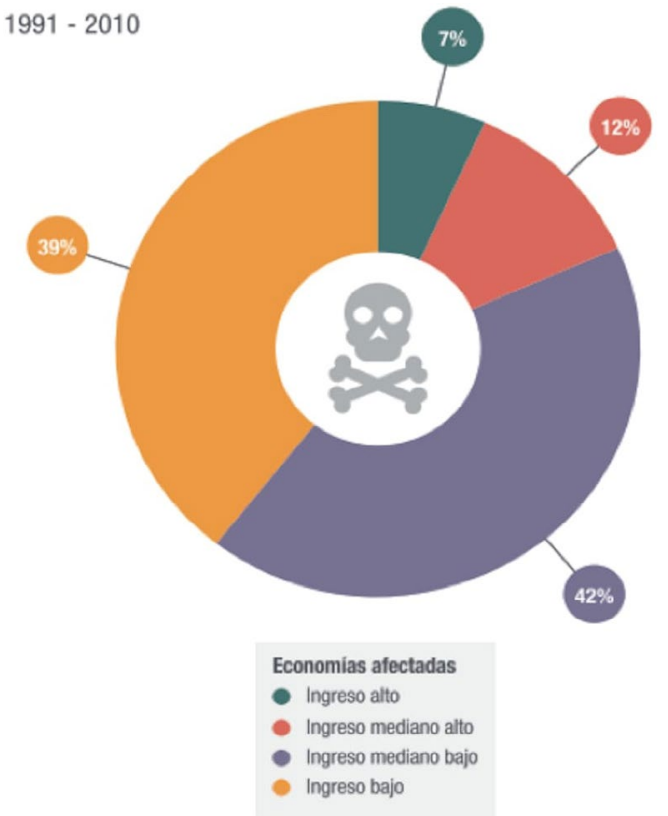

Figura 2. Muertes causadas por desastres de origen natural en el periodo 1991-2010 por tipo de economías afectadas Fuente: Watson et al. (2015)

La PF se ha asociado a la RR debido a que está ligada a medidas prospectivas de GRD, es decir, con la planificación y ejecución de medidas anticipadas que permitan reducir la probabilidad de ocurrencia de un desastre. La PF se enmarca en la RR en la medida en que aumenta la capacidad de los sistemas de soportar y recuperarse de impactos, en otras palabras, aumenta la resiliencia. En este sentido, es importante conocer las características de la PF y la dinámica de su implementación en América Latina, una región históricamente afectada por desastres.

El propósito de este estudio fue elaborar una caracterización general de la PF como medida de GRD en América Latina², a través de la presentación de los actores involucrados, los tipos de instrumentos más usados en la actualidad y algunos casos de estudio que pudiesen dar cuenta de los marcos regulatorios, avances y retos de la aplicación de dichos instrumentos. Se hace énfasis en la descripción de las características de los seguros indexados, debido al incremento importante en el uso de los mismos.

\section{CARACTERIZACIÓN DE LA PROTECCIÓN FINANCIERA}

La Protección Financiera (PF) se puede definir como el conjunto de mecanismos financieros de retención intencional o transferencia del riesgo que se establece de forma coordinada y planificada para acceder a recursos económicos oportunos y suficientes ante la potencial ocurrencia $u$ ocurrencia de un desastre (UNGRD, 2017; UNDRR, 2009). Según el Disaster Risk Financing and Insurance Program DRFIP (2020), la PF es benéfica para cuatro grupos: gobiernos nacionales y locales, propietarios de viviendas, pequeñas y medianas empresas, agricultores y la población más pobre.

La PF consta principalmente de dos componentes: la retención del riesgo y la transferencia del riesgo. La primera consiste en tomar medidas para el control del riesgo y asumir las pérdidas potenciales a través de un fondo (entre otros instrumentos) para que de forma planeada se pueda
1. Los 5 pilares de la GRD según DRFIP son: (i) Identificación del riesgo. (ii) Reducción del Riesgo. (iii) Preparación. (iv) Protección financiera. (v) Recuperación resiliente.

2. América Latina agrupa a los países cuya lengua oficial es el español el portugués a saber: Argentina, Bolivia, Brasil, Chile, Colombia, Costa Rica, Cuba, Ecuador, El Salvador, Guatemala, Honduras, México, Nicaragua, Panamá, Paraguay, Perú, Puerto Rico, República Dominicana, Uruguay y Venezuela. 
responder a las pérdidas causadas (Cardona, 2009). La segunda se puede definir como el proceso de trasladar de una parte a otra -formal o informalmente- las consecuencias financieras de un riesgo, mediante el cual un sistema obtendrá recursos de la otra parte después que se produzca un desastre (UNDRR, 2009).

Cuando se habla de PF nos referimos a esfuerzos sistémicos de reducción de la vulnerabilidad económica y por tanto, al aumento de la resiliencia financiera ante desastres; sin embargo, y de acuerdo con Miller y Keipi (2006), la PF por sí sola no constituye una estrategia de GRD, puesto que requiere de procesos simultáneos como el conocimiento, la reducción del riesgo y la recuperación.

Según GFDRR et al. (2014), la PF se encuentra constituida por cuatro estrategias fundamentales y la implementación de éstas le otorgan un enfoque integral en varios niveles territoriales y sectoriales, dichas estrategias son:

(a) Financiamiento soberano del riesgo de desastres. Los beneficiarios son los gobiernos y se caracteriza por incrementar la capacidad de respuesta y reconstrucción mediante la ejecución, la movilización de recursos/conocimientos y el aseguramiento de bienes públicos. Con esta estrategia, los gobiernos mejoran la estabilidad de las finanzas públicas al reducir incertidumbre en los efectos de los desastres y provee incentivos para la inversión en Reducción del Riesgo (RR).

(b) Aseguramiento de propiedades ante el riesgo de desastres. Los beneficiarios directos son los propietarios de inmuebles y las Pequeñas y Medianas Empresas (Pymes) puesto que la estrategia provee compensación por daño físico y compensaciones por afectaciones indirectas derivadas del daño.

(c) Seguro agrícola. Los beneficiarios de esta estrategia son los agricultores, los cuales reducen su vulnerabilidad mediante la compensación por daños en su producción y/o daños en sus bienes de producción. Asimismo, ayuda a la distribución de la carga en los procesos de recuperación entre el sector público y el privado, aumenta el entendimiento de la vulnerabilidad financiera por desastres y para productores de bajos ingresos, aumenta la competitividad y el acceso a servicios y mercados financieros.

(d) Protección social vinculada a desastres. Los principales beneficiarios son las personas más vulnerables económicamente. Esta estrategia dirigida a los más pobres mitiga los impactos derivados de desastres generando compensación en los medios de vida o bienes afectados a través de flexibles redes de seguridad social. Aumentando la resiliencia, se evita que las personas vulnerables caigan en situación de pobreza por el impacto de un desastre (GFDRR et al., 2014).

Podemos decir entonces que la PF puede ser aplicada por todos los actores de la sociedad, de forma individual o colectiva de acuerdo a sus necesidades. Ésta puede ser tomada por los gobiernos nacionales (para aseguramiento de bienes públicos/infraestructuras, sectores, población vulnerable, entre otros), gobiernos regionales y locales (aseguramiento de infraestructura pública y bienes públicos, población vulnerable y procesos de sectores estratégicos), propietarios de viviendas y otros inmuebles, agricultores y personas en condición de vulnerabilidad (con el apoyo de financiadores nacionales o extranjeros -donantes-, gobiernos locales, nacionales o subnacionales).

\begin{tabular}{|c|c|c|c|}
\cline { 2 - 4 } \multicolumn{1}{c|}{} & Impacto Bajo & $\begin{array}{c}\text { Impacto } \\
\text { Moderado }\end{array}$ & Impacto Alto \\
\hline $\begin{array}{c}\text { Recurrencia } \\
\text { Baja }\end{array}$ & $\begin{array}{c}\text { Fondos de mitigación, } \\
\text { los créditos y los } \\
\text { pagos directos }\end{array}$ & $\begin{array}{c}\text { Seguros y } \\
\text { reaseguros }\end{array}$ & $\begin{array}{c}\text { Bonos catastróficos, derivados } \\
\text { climáticos, créditos contingentes y } \\
\text { préstamos para reconstrucción }\end{array}$ \\
\hline $\begin{array}{c}\text { Recurrencia } \\
\text { Media }\end{array}$ & $\begin{array}{c}\text { Medidas de mitigación } \\
\text { del riesgo } \\
\text { estructurales y/o no } \\
\text { estructurales }\end{array}$ & $\begin{array}{c}\text { Seguros y } \\
\text { reaseguros }\end{array}$ & $\begin{array}{c}\text { Bonos catastróficos, derivados } \\
\text { climáticos, créditos contingentes y } \\
\text { préstamos para reconstrucción }\end{array}$ \\
\hline Alta & $\begin{array}{c}\text { Medidas de mitigación } \\
\text { del riesgo } \\
\text { estructurales y/o no } \\
\text { estructurales }\end{array}$ & $\begin{array}{c}\text { Seguros } \\
\text { catastróficos }\end{array}$ & $\begin{array}{c}\text { Medidas de adaptación e instrumentos } \\
\text { financieros como fondos de } \\
\text { prevención y desarrollo -retención del } \\
\text { riesgo-y créditos para prevención. }\end{array}$ \\
\hline
\end{tabular}

Figura 3. Matriz para selección de instrumentos de protección financiera según impacto y recurrencia de las amenazas naturales Fuente: Autor, 2020, a partir de Ministerio de Hacienda y Crédito Público de Colombia (2012) 
La creación o fortalecimiento de la resiliencia financiera en un territorio se puede implementar mediante acciones que reúnan las siguientes cinco características (GFDRR et al., 2014): (i) Apropiada información sobre el riesgo (Conocimiento del riesgo y comunicación); (ii) responsabilidad sobre el riesgo (gobiernos, civiles, empresas, donantes); (iii) costo del capital potencialmente afectado (evaluación del riesgo de desastre); (iv) puntualidad del financiamiento postdesastre y disciplina en la disposición de recursos (gobernabilidad), (v) la planificación y la vinculación multisectorial en la preparación y ejecución de la respuesta ante desastres.

Los instrumentos para usar se pueden identificar conociendo el impacto y recurrencia de las amenazas naturales. En la Figura 3 se presentan los instrumentos más apropiados de acuerdo a las características del riesgo.

Los instrumentos de PF deberían estar enmarcados en estrategias de protección financiera y éstas a su vez formuladas a la sombra de un plan territorial (nacional o municipal) o sectorial de GRD para garantizar la aplicación sistémica y articulada de una visión concertada de desarrollo.

\section{Principales actores}

Se pudieron identificar una serie de actores clave en la planificación, inversión y aplicación de la PF en América Latina. La identificación de los actores más relevantes en la región contribuye a la realización de un mapa de actores que aporta a la visibilidad y diálogo entre las partes interesadas.

Como organismos multilaterales se destaca el Banco Mundial, el Banco Interamericano de Desarrollo (BID), el Fondo Global para la Reducción del Riesgo de Desastres y la Recuperación (GFDRR en inglés), la Comunidad Andina de Naciones (CAN), La Organización para la Cooperación, el Desarrollo Económico (OCDE), la Asociación Internacional de Supervisores de Seguros (IAIS en inglés), la Corporación Andina de Fomento (CAF), la Iniciativa para el Acceso a Seguros (A2ii), la Red de Microseguros (MiN en inglés), El Fondo de Seguros contra Riesgos de Catástrofe en el Caribe (CCRIF en inglés), entre otros.

Las agencias de cooperación internacional más destacadas en la región son: Agencia de Cooperación Internacional de Japón (JICA en inglés), Agencia Suiza de Cooperación y Desarrollo (SECO), Corporación Alemana para la Cooperación Internacional (GIZ en alemán)/ el Banco de Desarrollo Alemán (KfW)/ Ministerio Federal de Cooperación Económica y Desarrollo de Alemania (BMZ), Agencia de los Estados Unidos para el Desarrollo Internacional (USAID en inglés) y el Departamento de Desarrollo Internacional del Reino Unido (DFID en inglés).

Se pudieron identificar importantes organizaciones privadas y públicas generadores de datos e información como Munich Reinsurance Company (Munich-Re), Hannover Re SE, Swiss Reinsurance Company Ltd. (Swiss Re), el Fondo para Desastres Naturales (NDF en inglés) y el Fondo de Inversión IsuResilience (IIF en inglés).

Se pudo conocer una participación concreta y activa en PF para la Gestión del Riesgo de Desastres (GRD) de los ministerios de finanzas de países como Brasil, Chile, Colombia, Costa Rica, Guatemala, México, Panamá, Perú, Puerto Rico, República Dominicana y Uruguay. Se destacan estos ministerios en la GRD puesto que su participación es fundamental para la ampliación de los roles al interior de los sistemas nacionales de GRD, compartiendo la responsabilidad de la GRD y la PF con otros actores nacionales de la gestión del desarrollo.

La revisión de la literatura, los casos de estudio y los nuevos marcos regulatorios permiten conocer una estrecha relación del sector privado y el público en el desarrollo de la PF en América Latina. Este hecho es bastante positivo si consideramos que el sector privado en general ha estado relativamente distante, a nivel local, de las acciones de GRD desarrolladas por el sector público.

\section{Marco regulatorio}

Debido a que en América Latina no se cuenta con un marco regulatorio común para la Protección Financiera (PF), se optó en el presente estudio por mencionar avances en el desarrollo de las políticas públicas de GRD que consideran explícitamente a la PF. En este sentido, se presentan a continuación tres casos de estudio (México, Colombia y Perú) que dan cuenta de los avances en esta materia.

En México se cuenta con la Estrategia Financiera de Administración de Riesgos de Desastres que responde a la evolución del enfoque en el manejo de los riesgos del país, así, la estrategia incluye la retención del riesgo (FONDEN \& FOPREDEN), la transferencia del riesgo y la reconstrucción. La 
estrategia cuenta con un enfoque innovador en el sentido de que incluye explícitamente acciones ex-ante -acciones ligadas a la prevención- y ex-post -acciones de respuesta, recuperación y reconstrucción- (Secretaría de Hacienda y Crédito Público de México, 2012).

La estrategia contempla una cuantificación del riesgo que determina el instrumento de retención o transferencia a aplicar. Con base en el conocimiento del riesgo se desarrollan medidas de prevención, de recuperación (basado en un programa de reubicación) y auxilio o respuesta a desastres. La contratación de las coberturas se basa en la protección a infraestructura federal y estatal (incluyendo vivienda en pobreza patrimonial) (Ídem).

En Colombia la regulación de la PF presentó un avance significativo cuando en 2012 la Ley 1523, transforma el Fondo Nacional de Calamidades en el Fondo Nacional de Gestión del Riesgo de Desastres, que incluye un enfoque integral de la Gestión del Riesgo de Desastres (GRD) al considerar en dicho fondo cinco cuentas a saber: (i) subcuenta para el conocimiento del riesgo, (ii) para la reducción del riesgo, (iii) para el manejo de desastres, (iv) para la protección financiera, (v) subcuenta para la reconstrucción. La subcuenta de PF fue atribuida al Ministerio de Hacienda y Crédito público de Colombia (MHCP) para el desarrollo de la Estrategia Financiera ante Eventos de Origen Natural. Otro beneficio destacable de la Ley 1523 del 2012 es que incluye al MHCP como parte del Consejo Nacional de Gestión del Riesgo de Desastres, crea el Comité Nacional para la Reducción del Riesgo que, a su vez, considera como uno de sus miembros a la Asociación de Aseguradores Colombianos (FASECOLDA).

Posteriormente, el Gobierno de Colombia con el apoyo del Banco Mundial, desarrollaron la "Estrategia de política de gestión financiera pública ante el riesgo de desastres por fenómenos de la naturaleza" que establece tres objetivos marco para el desarrollo de la PF en el país: (1) Identificación y entendimiento del riesgo fiscal de los desastres; (2) Gestión financiera de los desastres; (3) Aseguramiento del riesgo catastrófico de los activos (Banco Mundial, 2014). Esta estrategia se enmarca en el Plan Nacional de GRD de Colombia, con un horizonte de tiempo de 10 años (2015-2025). Se destacan los avances que el sector asegurador ha fomentado en los últimos 10 años mediante el desarrollo de microseguros, especialmente la innovación en los canales de distribución de los productos. En este mismo sentido, Colombia mediante FASECOLDA ha impulsado diferentes estrategias que buscan incentivar la creación de oferta en seguros para las poblaciones de bajos ingresos. (Camargo et al., 2014).

En Perú se destaca la creación del Fondo de Estabilización Fiscal (FEF) instituido en el año 1999 como medida de retención del riesgo para responder a emergencias y acciones de recuperación y reconstrucción postdesastre. En 2011 se creó un sistema para la implementación de la PF en la GRD, representado por la Dirección de Gestión del Riesgo que a su vez hace parte del Ministerio de Economía y Finanzas (MEF). La dirección como órgano coordinador tiene la finalidad de gestionar los riesgos financieros, operativos y contingentes de naturaleza jurídica y contractual o derivados de desastres, así como de acciones para su gestión. En 2012, el país avanzó mediante la conformación del Comité de Riesgos presidido por el MEF; esta importante instancia contiene un grupo de trabajo para la articulación financiera postdesastre de gran magnitud (GFDRR et al., 2016). En 2016 fue publicada la Estrategia Integral de protección financiera ante el riesgo de desastres asociados a fenómenos naturales del Perú (GFDRR et al., 2016) la cual establece las líneas estratégicas de intervención en PF ante desastres de origen natural.

Según Camargo y Gonçalves (2014), Perú se encontró por seis años consecutivos como primero en el ranking de los países de la región, con mayor desarrollo legal y regulatorio del sector de microfinanzas. En términos regulatorios, se pudo conocer una fuerte actividad del sector privado en el posicionamiento e implementación de los microseguros ${ }^{3}$ enfocados a la población de bajos ingresos (que en Perú constituye el 37,9\%). Los microseguros se encuentran regulados por la Resolución SBS 14283 - 2009. Entre otros instrumentos regulatorios específicos, se resaltan las resoluciones SBS 2996 de 2010 -relativo a la comercialización de productos de seguro-, SBS 1797 de 2011 -sobre el registro de intermediarios y asistentes de seguros-, la SBS 3198 de 2013 regulación sobre el pago de las primas-, SBS 3199 de 2013 -sobre transparencia de la información y contratos de seguro-, la SBS 3202 de 2013 -sobre la gestión de reclamaciones y pagos- y la SBS 3201 de 2013 -sobre el uso de pólizas electrónicas- (Camargo \& Gonçalves, 2014).

\section{Tipos de instrumentos}

La Protección Financiera (PF) presenta varios tipos de instrumentos para hacer frente a desastres asociados a fenómenos naturales. Según Keipi et al. (2002) y Ministerio de Hacienda y

3. Para 2014, Access to Insurance Initiative (Azii) estimó que el mercado potencial de microseguros en Perú representaba el $50 \%$ de la población total del país. 
Crédito Público de Colombia (2012), los instrumentos más usados en la región son:

Fondos de Reserva: Es una medida de retención del riesgo que tiene como objetivo ser la fuente de liquidez luego de la ocurrencia de un desastre. La ventaja de la implementación de estos fondos es que se evitan los traslados presupuestales que tanto frenan el desarrollo de los objetivos de las entidades gubernamentales tras una situación de desastre. El reto de su implementación es la evaluación de los riesgos y estimación de pérdidas probables para garantizar fondos suficientes para la respuesta, recuperación y reconstrucción.

Créditos Contingentes: Estos créditos son pre-aprobados y desembolsados rápidamente ante la ocurrencia del desastre. El beneficio de este instrumento es que actualmente se ofrece a tasas de interés inferiores a las de los créditos convencionales, por otra parte, para conservar el derecho de acceder a esos fondos el prestatario paga una tasa de manejo administrativa (Ministerio de Hacienda y Crédito Público de Colombia, 2012). Adicionalmente, los pagos de intereses solo comienzan cuando se accede al crédito para manejar el desastre (Miller \& Keipi, 2006). Estos instrumentos no transfieren el riesgo, puesto que es responsabilidad del estado la reconstrucción.

Títulos valores: Según Miller y Keipi (2006), los niveles más altos de riesgo de desastres pueden ser gestionados utilizando títulos valores provistos por los mercados internacionales de capital. Dos de los títulos más conocidos en el mercado son los 'bonos de catástrofe' y los 'derivados climáticos'.

Al ser un instrumento de retención del riesgo, los bonos de catástrofe (conocidos como CAT) permiten al emisor (poseedor del riesgo) transferir el riesgo al mercado de capitales. Estos son emitidos por la entidad en riesgo con un horizonte de tiempo de entre tres a cinco años. Los bonos son calificados por agencias independientes en el marco de la calificación del crédito, para esto se tiene como base los resultados de los modelos y los análisis del riesgo. Pueden ser paramétricos (con un índice de pérdidas) o basados en las pérdidas.

Los bonos CAT han representado un instrumento importante de PF en América Latina, por ejemplo, en 2008 el Banco Mundial realizó la primera aprobación de un préstamo a Costa Rica con una opción de reducción diferida por catástrofe (conocida como CAT-DDO). El Banco Mundial ha aprobado CAT-DDO en varios países de la región, estos préstamos incluyen (en US\$): \$150 millones a Colombia en 2008 y $\$ 250$ millones en 2011; $\$ 50$ millones a El Salvador en 2011; $\$ 66$ millones a Panamá en 2011; \$100 millones a Perú en 2010; \$85 millones a Guatemala en 2009; y \$65 millones a Costa Rica en 2008 (GFDRR et al., 2014).

Otras organizaciones internacionales han comenzado a ofrecer crédito contingente como un producto de financiamiento de riesgo de desastres que no solo aumenta la capacidad de recuperación financiera, sino que ayuda a incentivar mejores políticas de GRD en general (GFDRR et al., 2014). Es importante mencionar que los créditos contingentes no solo son usados para la respuesta y la recuperación a desastres, sino que pueden ser usados para la creación y fortalecimiento de políticas e instrumentos de planificación para la respuesta, recuperación y reconstrucción.

Los derivados climáticos están destinados a la protección ante riesgos del régimen climático como las variaciones extremas en temperaturas, lluvias, vientos, etc. $y$, son usados para cubrir riesgos con alta probabilidad de ocurrencia y bajo impacto en las afectaciones. Son un buen complemento a los bonos CAT o a los seguros contra desastres por su relación con eventos de alta probabilidad y bajo impacto

Cautivas: Es una empresa de seguros o reaseguros, según la naturaleza de su actividad, que tiene por objetivo asegurar o reasegurar riesgos y se encuentra constituida por un grupo económico para beneficio de las empresas que conforman el mismo, constituida de conformidad con una legislación extranjera desde donde opera (Cardona, 2009). El respaldo financiero de las empresas cautivas proviene de las cuotas de participación de cada país que es igual a la prima anual que paga cada país por la cobertura de riesgos. (GFDRR et al., 2014)

Seguros: Son una medida de transferencia del riesgo que, a diferencia de los instrumentos de retención del riesgo, elimina el costo de oportunidad de mantener recursos en una cuenta, de esta forma, para acceder al seguro solo se paga de forma periódica una prima del valor del riesgo. Por otra parte, en cuanto más partes compartan el riesgo (sectores, gobiernos, población, etc.) más se reducirán los costos de implementación de un seguro (Banco Mundial, 2014).

Las desventajas más significativas en la implementación de los seguros son: Los pagos (primas) se pierden ${ }^{4}$ si no se produce un desastre, se puede producir selección adversa, riesgo no cubre el $100 \%$ de las pérdidas, es igualmente benéfico realizar acciones complementarias para que el evento peligroso no se presente y así reducir el riesgo a niveles tolerables. 
base, riesgo moral y se debe incurrir en el costo de transacción -estos términos son ampliamente descritos por Miller y Keipi (2006).

Seguro Indexado: Para efectos de este artículo, los seguros indexados serán descritos con mayor detalle, debido a la importancia que han tomado en América Latina y por el notorio componente innovador y de rápida evolución que tienen en el mundo.

¿Cómo funciona? El seguro indexado paga beneficios sobre la base de un índice predeterminado de acuerdo con niveles presentados durante la manifestación del fenómeno amenazante. El pago se realiza por pérdida de activos e inversiones que representa un capital de trabajo para el asegurado, no solo en las pérdidas directas sino por pérdidas relacionadas con interrupciones en su proceso de producción.

Al no requerir un asesor de reclamo de seguros tras un evento (es decir que no requieren la evaluación de pérdidas individuales), como ocurre con los seguros tradicionales, el seguro indexado permite que el proceso de liquidación de reclamos sea más rápido y transparente (GFDRR et al., 2014)

El seguro indexado depende de la disponibilidad de datos, por ejemplo, se puede usar un índice paramétrico cuando solo se tienen datos disponibles de la amenaza y de esta forma, el seguro solo pagaría el evento amenazante dado. Por otra parte, se puede adaptar a un índice de pérdida cuando existe información de los bienes expuestos, de esta forma, el seguro paga de acuerdo a la pérdida modelada con base en la información de exposición y de acuerdo a los registros en los parámetros físicos del evento. En este sentido, el desarrollo de seguros indexados exige avances en el monitoreo de los fenómenos amenazantes y una articulación entre las agencias internacionales, los gobiernos nacionales y locales y el sector asegurador.

Para la determinación de un índice (umbral) se acude a información de instituciones independientes oficialmente reconocidas, estas instituciones proveen los valores de los parámetros del fenómeno amenazante en cuestión. El desembolso está soportado por fuentes independientes verificables y componentes paramétricos claros, el pago previamente acordado se hace de manera rápida, sencilla y sin largos ajustes (Lemke, 2019).

GFDRR et al. (2014) mencionan que las ventajas que hacen diferenciable al seguro indexado del seguro tradicional o de indemnizaciones, es que permite pagos más rápidos, menores costos administrativos, menor riesgo moral y selección adversa (términos que serán desarrollados más adelante). De igual forma, A2ii e IAIS (2018) refieren que la ventaja más destacable de los seguros indexados como medida de reducción del riesgo de desastres, está en que los asegurados no tienen que formular una solicitud de compensación, puesto que es responsabilidad de la aseguradora informar a los asegurados que el índice alcanzó el umbral y que los pagos se realizarán. El deber ser en este caso es que la aseguradora verifique los pagos, los comunique y los haga efectivos en un plazo predefinido en días.

Una desventaja común de los seguros indexados es el desafío que presenta frente a la disponibilidad y precisión de los datos y, por tanto, de los modelos (conocido como riesgo base). Esto para garantizar que las pérdidas individuales coincidan con la medición del índice, puesto que de esta forma se puede reducir el riesgo de que un asegurado sufra un desastre sin que el índice alcance el umbral predeterminado y que no se genere un pago. Contrariamente, un pago podría activarse sin ningún daño o pérdida incurrida. Para cualquier gobierno es crucial comprender el riesgo base dadas las opciones de seguro propuestas, y llevar a cabo un análisis de costo-beneficio de diferentes índices potenciales con diferentes niveles de riesgo base, procurando el producto que mejor se adecúe tanto a las características de los beneficiarios como a la disponibilidad de la información y confiablidad de los modelos (GFDRR et al., 2014).

Microseguros: También llamados 'seguros inclusivos', son un importante segmento del crecimiento de seguros en América Latina, desarrollados específicamente para la población de bajos ingresos, asequibles y gestionados con base en principios de riesgo. McCord et al. (2015) llama 'Consumidores emergentes' al mercado de microseguros en América Latina y hacen énfasis en que los microseguros deben ser especialmente diseñados para los más pobres y vulnerables; dichos autores insisten en que el diseño de microseguros no se debe limitar a productos de bajo costo que brinden acceso a personas de bajos ingresos, sino que deben estar pensados de acuerdo a necesidades específicas, en otras palabras, productos que incluyan el enfoque diferencial en los territorios. 


\section{RETOS Y AVANCES EN AMÉRICA LATINA}

Es necesario considerar como un reto la investigación sobre las limitantes que presentan los mercados de América Latina, es decir, ¿qué factores internos y/o externos llevan a restringir la adquisición de instrumentos de PF, especialmente seguros para desastres? A simple vista, pareciera ser un mero asunto de capacidad económica, sin embargo, enfoques conceptuales sobre la reducción del riesgo y la atención de los desastres por parte de los gobiernos puede jugar un papel fundamental en el desarrollo de un mercado. Esta limitante es desarrollada ampliamente por Raschky y Weck-Hannemann (2007), quienes mediante el análisis del término 'Charity Hazard', asocian el enfoque gubernamental centrado en la atención de los desastres a través de medidas paternalistas, con la desmotivación de los individuos para implementar medidas de transferencia del riesgo. Esto representa un problema en la medida en que las personas no implementan la PF porque esperan una asistencia externa total.

El Charity Hazard representa un factor limitante debido a que es altamente compleja su gestión en la práctica. Este fenómeno no obedece a razones técnicas o a las características de los desastres, sino que según Garrett y Sobel (2003) implica intereses políticos de obtención de visibilidad y, en ocasiones se enmarcan en estrategias de reelección de grupos políticos (Schwarze \& Wagner, 2004).

\section{Retos}

A continuación, se presentan algunos retos por tipo de actor que son actualmente reconocidos como determinantes para el desarrollo de la PF en la región:

Gobiernos nacionales y locales:

- Generar análisis de la efectividad de las medidas de PF en la reducción de las condiciones de riesgo a escala local y nacional. Para mejorar esta necesidad, El Banco Mundial ha desarrollado el Programa de Evaluación de Impacto, acompañado de una guía detallada para tal efecto (Gertler et al., 2011).

- Incentivar el desarrollo de mercados diferenciados de acuerdo a las dinámicas sociales y económicas de los países. Lo anterior debido a que según KfW Development Bank \& InsuResilience Global Partnership (2019), el 90\% de las pérdidas registradas en América Latina, derivadas de desastres del régimen climático no estaban aseguradas, mientras que en países de ingresos altos el $50 \%$ de las pérdidas están cubiertas por seguros.

- Implementar un marco normativo claro de GRD en donde se establezcan las responsabilidades por los daños financieros del gobierno nacional y de los gobiernos subnacionales.

- Para evitar que el riesgo siga creciendo hasta el punto en el que no sea posible su aseguramiento, los gobiernos deben trabajar en la regulación de los mercados de seguros y el control de las zonas de riesgo para evitar la falla del mercado (Geneva Association, 2013).

Gobiernos nacionales/locales, sector privado y agencias internacionales de monitoreo:

- Expansión, difusión y comunicación de datos y tecnología para el mejoramiento de alertas tempranas ante eventos climáticos extremos que puedan impactar la economía, los bienes y medios de subsistencia y las personas.

- Realización de encuentros periódicos para discusión y comunicación de retos, dificultades y avances en el desarrollo de nuevas tecnologías y enfoques.

- Promover la Gestión del Riesgo de Desastres (GRD) con enfoques de intervención innovadores en regiones vulnerables (Miles \& Wiedmaier-Pfister, 2019).

- Articulación sector privado y público: sus beneficios no se limitan a la implementación de una estrategia o instrumentos de PF, sino que reduce la vulnerabilidad institucional de los territorios, en la medida en que fortalece las capacidades técnicas y administrativas del sector público mediante el incentivo y fortalecimiento de estándares técnicos y de la transparencia en la gestión de las finanzas públicas.

Con la adopción de términos basados en estándares internacionales para contratos de seguros, los gobiernos pueden llevar estas prácticas internacionales a los mercados de seguros nacionales, incentivando el mejoramiento de los procedimientos y productos brindados por el sector en el país. 
Por ejemplo, en Colombia, el gobierno utiliza términos estandarizados de las mejores prácticas del mercado internacional de seguros para comprar un seguro contra catástrofes para sus edificios públicos. En México en el año 2011, para poder colocar un contrato de seguro por exceso de pérdida basado en indemnización en los mercados privados, fue necesario que el gobierno desarrollara procesos transparentes y sólidos para la presentación de informes de pérdidas (Marulanda et al., 2014)

Gobiernos nacionales, locales, sector privado, agencias de cooperación internacional e Organismos multilaterales:

- Entablar un diálogo en el diseño de instrumentos que permitan cerrar la brecha de la protección. Según Ghesquiere y Mahul (2010) se deben diseñar estrategias integrales territoriales de PF en lugar de centrarse en productos individuales.

- Desarrollo de enfoques inclusivos diferenciales y participativos, que involucren a jóvenes, grupos indígenas, comunidades locales y mujeres, durante el proceso de desarrollo de las soluciones (instrumentos/productos).

Sector privado relacionado con la protección financiera:

- Disolución del cariz competitivo en el sector y el fomento de acciones colaborativas y complementarias entre las instituciones.

- Posicionamiento de la asistencia técnica a los gobiernos nacionales y locales como valores agregados de sus productos.

- McCord et al. (2015) aseguran que la tecnología de fácil acceso es determinante para la aplicación y difusión de tecnologías que reduzcan los costos y aceleren los procesos; por ejemplo, el uso de teléfonos móviles ha representado una herramienta de gran ayuda al desarrollo de microseguros. Igualmente, Leach (2012), menciona que los beneficios de la aplicación de la tecnología en los microseguros se pueden resumir en seis componentes: reducción de los costos de administración; alcance de nuevos mercados; enlaces a diferentes operaciones de manera simultánea; mejoramiento del servicio al cliente; reducción del fraude; y mejor entendimiento de los mercados.

En América Latina reconocer de forma diferencial la vulnerabilidad social, se sitúa como una de las prioridades más notorias para gobiernos y sector privado (Miles, K. Wiedmaier-Pfister, 2018). Miles y Wiedmaier-Pfister (2019), aseguran que la mujer se encuentra en el foco del desarrollo de procesos de diseño de productos de PF como una de las medidas de reducción de la vulnerabilidad en el marco de la GRD.

Otro desafío transversal se refiere al riesgo moral, que compete gestionar a todos los actores relacionados con la PF y en general con la GRD, puesto que los instrumentos financieros no pueden resolver ni atender todas las necesidades relacionadas con el riesgo y los desastres, es necesario generar consciencia de la importancia de generar conocimiento, de reducir el riesgo y prepararse para responder a un desastre, aunque se cuente con suficientes medidas de PF.

Difundir las ventajas del pago transparente de las reclamaciones realizadas por los clientes tras una situación de desastre contribuye con el progreso de los seguros y otros instrumentos de $P F$, esto porque se dinamiza la construcción de mercados pues se aumenta la visibilidad de las aseguradoras y la confianza en la efectividad de los seguros. Por ejemplo, McCord et al. (2015, p.25) afirman que, en el caso de los microseguros considerados como "Una industria donde es un obstáculo el desconocimiento de los productos por parte de los clientes de bajos ingresos", la capacidad de pagar más reclamaciones (mientras se mantiene una utilidad justa) es un claro ganar-ganar.

Los retos actuales de los seguros incluyentes o microseguros en América Latina se pueden agrupar en cuatro: (i). Articulación entre el sector público y el sector privado; (ii). Disponibilidad, acceso y confiabilidad de los datos y modelos; (iii). Implementación de políticas públicas y marcos regulatorios; (iv). Diseño y acceso a los productos para grupos vulnerables.

Finalmente, las lecciones aprendidas tras la aplicación de microseguros basados en índices en Guatemala, presentadas por Access to Insurance Iniciative (Aii) \& International Association of Insurance Supervisor IAIS (2018) pueden resumir claramente gran parte de los retos planteados en el presente acápite. A continuación, se mencionan de forma general dichos retos: (i) Creación de consciencia de la necesidad de buscar alternativas para los segmentos más vulnerables de la 
población. (ii) Prevalencia del principio de indemnización y pago de reclamaciones. (iii) Programas constantes de educación financiera. (iv) El asegurado debe comprender el producto puesto que de esta forma se aumenta la confianza en la manera en que opera el producto. (v) Transparencia de los procedimientos de pago de beneficios. (vi) Protección del consumidor. (vii) Implementación de tecnología de fácil acceso.

\section{Avances}

Se destacan avances que integran esfuerzos internacionales como el Fondo Global de Seguros Indexados (GIIF), establecido en 2009 como un fondo fiduciario de múltiples donantes operado por el Instituto de Finanzas Corporativas (CFI) y el Banco Mundial. Este fondo tiene por objetivo fortalecer el crecimiento de los mercados locales para seguros basados en índices climáticos y desastres; ha sido destinado a países en desarrollo de Asia Pacífico, África, América Latina y el Caribe.

La consideración de las diferencias sociales en el desarrollo de productos de PF es otro gran avance, muestra de esto son los seguros, por ejemplo, la experiencia de la Organización de Riesgos de Catástrofes de Microseguros (MiCRO), creada en 2011 como una asociación públicoprivada con fondos de capital público donado para proporcionar cobertura de microseguros a las microempresas de Haití, propiedad de mujeres y con considerables niveles de vulnerabilidad (Swiss Re, 2020). MiCRO ha desarrollado esquemas de seguros en América Latina que responden a problemas identificados en comunidades indígenas y de género (acceso y empoderamiento de la mujer); contribuyendo de una manera más específica con productos especialmente diseñados para los más vulnerables (Miles \& Wiedmaier-Pfister, 2019).

En aras de ejemplificar los avances de la PF en América Latina, a continuación, se presentan tres casos de estudio: México, Colombia y Perú. Estos tres países presentan características no muy disímiles en términos de amenazas y vulnerabilidades; sin embargo, sí presentan diferencias en la experiencia de la implementación de la PF para la Gestión del Riesgo de Desastres (GRD).

México: Muestra de la experiencia sólida del país en la PF data de 2006, cuando transfirió a los mercados financieros $\$ 450$ millones de riesgo por sismo. Esta transferencia del riesgo se dio mediante la combinación del primer bono gubernamental de catástrofe en el mundo (CAT) con un seguro paramétrico (con valores de $\$ 160$ millones y $\$ 290$ millones respectivamente) (Secretaría de Hacienda y Crédito Público de México, 2012).

El gobierno de México estableció un mecanismo de notificación de pérdidas posteriores a desastres administrado por su Fondo de Desastres Naturales (FONDEN), que permite a los estados afectados acceder a pagos puntuales directamente desde dicho fondo, reduciendo los problemas de coordinación que requieren mucho tiempo. EI FONDEN ha evolucionado traspasando la frontera de la respuesta y reconstrucción postdesastre para la destinación de recursos para inversión en reducción del riesgo, conocimiento de las amenazas y la comunicación; de esta forma, promueve la gobernanza y la transparencia al exigir que los gobiernos estatales completen una evaluación de riesgos antes de ser seleccionados para la financiación de proyectos de reducción del riesgo.

En el año 2009 y con una vigencia de tres años, el gobierno implementó un bono de catástrofe a través del MultiCat, destinado para la transferencia del riesgo ante sismo y ciclones tropicales. El bono se compuso de cuatro fases, cada fase destinada para un área geográfica diferente. Por ejemplo, la fase destinada para sismos es paramétrica, es decir, que se activa cuando se exceden los valores de magnitud establecidos para el sismo y la fase de ciclones tropicales se activa cuando la presión en el centro de la tormenta es igual o inferior a un umbral predefinido (Secretaría de Hacienda y Crédito Público de México, 2012)

Avances significativos en el componente técnico de modelación fueron los desarrollados en el marco del R-FONDEN, dedicado a la evaluación probabilista del riesgo de desastres con estimación de pérdidas de vidas y bienes materiales.

Colombia: En el año 2008, el país contrató con el Banco Mundial una Opción de Desembolso Diferido para Desastres de origen natural mediante un crédito contingente -CATDDO- por US\$ 150 millones. Siendo de libre destinación, el CATDDO fue utilizado por el gobierno para la atención y recuperación del desastre ocasionado por el fenómeno La Niña en 2010 (Ministerio de Hacienda y Crédito Público de Colombia, 2012).

En el año 2011, el gobierno incluyó la evaluación de pasivos contingentes por desastres de origen no intencional en la estrategia de gestión de riesgos fiscales. En consecuencia, a partir del 
año 2012, en el inicio de la implementación de la estrategia de PF, el gobierno utiliza términos y condiciones estandarizados informados por las mejores prácticas internacionales para comprar un seguro contra catástrofes para sus edificios públicos. Un caso concreto de aplicación de PF en Colombia ha sido el municipio de Manizales, departamento de Caldas, que, mediante el sistema de impuestos a la propiedad, ha conseguido pagar primas para la implementación de un seguro colectivo voluntario ante sismos (Marulanda et al., 2014).

Colombia, siguiendo el liderazgo de la industria de seguros y sin un marco regulatorio específico, ha logrado un progreso significativo en la agenda para mejorar el acceso al seguro por parte de todas las personas, gracias a la oferta de seguros masivos de bajo costo (Camargo et al., 2014); sin embargo, la oferta de seguros de bajo costo debe dirigirse hacia el reconocimiento de las necesidades de diferenciadas de la población de bajos ingresos, para lo cual se apela a los microseguros o seguros inclusivos.

Perú: Los avances se evidencian en la integralidad de los planes (como el PLANAGERD ${ }^{5} 2014$ 2021 publicado mediante Decreto Supremo D.S. 034-2014-PCM) y las estrategias diseñadas para la implementación de la Gestión del Riesgo de Desastres (GRD). Muestra de esto, son las seis líneas prioritarias de intervención establecidas por el Ministerio de Economía y Finanzas del Perú (MEF) en el marco de la Estrategia Integral de Protección Financiera (EIPFRD) ante el Riesgo de Desastres Asociados a Fenómenos Naturales. En dicha estrategia se presentan las líneas de intervención priorizadas. Esta estrategia incluye un fuerte énfasis en el seguro de activos públicos y la mejora de la capacidad de gestión del riesgo ante sismos de las aseguradoras peruanas.

Las líneas de intervención de dicha estrategia están dirigidas a desastres derivados de fenómenos naturales, sus características principales son: (i) Identificar, cuantificar y evaluar el riesgo fiscal de los desastres. (ii) Formular los componentes para el desarrollo e implementación de herramientas de PF. (iii) Establecer lineamientos para el uso de los fondos disponibles para la atención post desastres. (iv) Promover la estimación, prevención, reducción del riesgo y preparación de la respuesta a desastres a través de mecanismos financieros e incorporar la GRD en la inversión pública. (v) Promover el desarrollo del mercado doméstico de seguros catastróficos ante amenazas naturales. (vi) Articular y promover la gestión de la continuidad operativa del Estado para la implementación de la estrategia financiera en el marco de la GRD (GFDRR et al., 2016).

En el año 2013 se aprobó el financiamiento contingente por un monto de US\$300 millones con el BID, enfocado a la atención de desastres causados por sismos. En ese mismo año, se firmó con la Corporación Andina de Fomento (CAF) un crédito contingente no comprometido por un valor de US\$300 millones destinados al manejo de desastres de origen natural, específicamente para actividades de rehabilitación de servicios públicos y estudios previos para la reconstrucción de infraestructura pública en el marco de los desastres. Posteriormente, en 2014 se aprobó un crédito contingente con la Agencia de Cooperación Internacional del Japón (JICA) por un monto cercano a los US\$ 83 millones para financiar actividades de rehabilitación en caso de la ocurrencia de un desastre de grandes magnitudes de origen natural (GFDRR et al., 2016).

Par el año 2016 el MEF firmó con el Banco Mundial dos préstamos contingentes de US\$ 1.250 millones cada uno para la atención y recuperación en caso de ocurrencia de un desastre de origen natural o crisis económica o financiera. Estos dos préstamos se enmarcan en la estrategia mencionada y que actualmente está siendo implementada por el Gobierno del Perú.

\section{CONCLUSIONES}

Los principales actores que desarrollan la PF en América Latina son organismos multilaterales, agencias de cooperación internacional, tanto agencias nacionales como empresas privadas generadoras de datos y los ministerios de finanzas de los gobiernos nacionales.

Pese a que la PF se ha implementado por más de 20 años y a que su uso aumenta rápidamente (especialmente los seguros indexados), la región no cuenta con un marco regulatorio común y pocos países cuentan con estrategia de PF.

Actualmente, los retos de la PF y en especial de los seguros, se centran en tres aspectos: (i) Articulación entre el sector público y privado y, creación o fortalecimiento de marcos regulatorios; (ii) Mejoramiento y apertura de datos, modelos e información; (iii) Creación de estrategias y productos especialmente diseñados para los más pobres y grupos en condición de vulnerabilidad.

El presente estudio contribuye para la caracterización de la PF en el marco de la Gestión del Riesgo de Desastres (GRD) en América Latina y El Caribe, un territorio altamente diverso y
5. Establece como objetivos promover la transferencia del riesgo, incluyendo acciones para el desarrollo y mejora de los seguros contra riesgo de desastres tanto para las entidades públicas y las concesiones, como para los privados (particulares y empresas). 
dinámico. Esta investigación da a conocer de manera general el estado del arte en la región en aras de contribuir a la comunicación de medidas cada vez más usadas y viables para reducir las condiciones de riesgo actuales y futuras, en pro de territorios menos vulnerables.

Esta investigación alienta nuevos estudios que amplíen el conocimiento sobre el impacto de la PF en países de la región, acciones dirigidas a reducir el riesgo moral, caracterización de los seguros indexados a través la revisión de casos de estudio y enfoques de diferenciales para el diseño de instrumentos de PF a escala local.

\section{REFERENCIAS}

Access to Insurance Iniciative (Aii) \& International Association of Insurance Supervisor (IAIS). (2018). Situación de los Seguros Paramétricos y Retos para la Regulación. Retrieved from https://azii.org/ sites/default/files/reports/cc26 index insurance web sp.pdf

Asamblea Legislativa Plurinacional de Bolivia. (2014). Ley 602 de 2014: Por la cual se crea la Ley de Gestión de Riesgos. La Paz: Gobierno de Bolivia.

Banco Mundial. (2014). Colombia: Estrategia de política de gestión financiera pública ante el riesgo de desastres por fenómenos de la naturaleza. Bogotá: Banco Mundial.

Bello, O.D. (2017). Desastres, crecimiento económico y respuesta fiscal en los países de América Latina y el Caribe, 1972-2010. Revista de La CEPAL, 121, 8-30. https://repositorio.cepal.org/handle/11362/41141

Camargo, A. \& Gonçalves, L. (2014). Encouraging access to insurance in Peru: Reshaping the insurance environment for the underserved and unserved population. Lima: Aii.

Camargo, A., Tatin-jaleran, C. \& Gonçalves, L.G.F. (2014). Colombia: Toward an inclusive universal insurance sector. Retrieved from https://azii.org/en/knowledge-center/colombia-countrydiagnostic-making-insurance-markets-work-for-the-poor-microinsurance-policy-regulation-andsupervision-colombian-case-study

CAN. (2009). Atlas de las dinámicas del territorio andino: Población y bienes expuestos a amenazas naturales. In A. Velásquez (Ed.), Predecan. Cali: CAN.

Cardona, O.D. (2009). La gestión financiera del riesgo de desastres, instrumentos financieros de retención y transferencia para la Comunidad Andina. Lima: Comunidad Andina.

Leach, J. (2012). Protecting the Poor: A Microinsurance Compendium. Enterprise Development and Microfinance, 19(3). https://doi.org/10.3362/1755-1986.2008.022

Congreso de Colombia. (2012). Ley 1523: Por la cual se establece la Política Nacional para la Gestión del Riesgo de Desastres. Bogotá: Congreso de Colombia.

Congreso de la República del Perú. (2011). Ley 29664: Crea el Sistema Nacional de Gestión del Riesgo de Desastres. Lima: CRP.

Disaster Risk Financing and Insurance Program DRFIP. (2020). Tools And Principles Of Financial Protection. Retrieved from https://www.financialprotectionforum.org/third-party/microsite 1/ subpageo2.html

Garrett, T.A. \& Sobel, R.S. (2003). The Political Economy of FEMA Disaster Payments. Economic Inquiry, 41(3), 496-509. http://doi.org/10.1093/ei/cbgo23

Geneva Association. (2013). Warming of the Oceans and Implications for the (Re)insurance Industry. Ginerba: The Geneva Association.

Gertler, P., Martínez, S., Premand, P., Rawlings, L. \& Vermeersch, C. (2011). La evaluación de impacto en la práctica. Washington DC: The World Bank. https://doi.org/10.1596/978-0-8213-8541-8

GFDRR, Banco Mundial, Ministerio de Economia y Finanzas del Perú, Confederación Suiza. (2016). Perú: Estrategia Integral de protección financiera ante el riesgo de desastres asociados a fenómenos naturales. Washington DC: GFDRR.

GFDRR, The World Bank \& Swiss Confederation. (2014). A Financial protection against natural disasters: Form products to comprehensives strategies. An Operational Framework for Disaster Risk Financing and Insurance. Washington DC: The World Bank. Retrieved from https:/gfdrr.org/sites/ gfdrr/files/publication/Financial Protection Against Natural Disasters.pdf

Ghesquiere, F. \& Mahul, O. (2010). Financial Protection of the State against Natural Disasters A Primer. The World Bank Policy Research Working Paper, 5429, 1-26.

Keipi, K. \& Tyson, J.(2002). Planificación y protección financiera para sobrevivir los desastres. Washington DC: Banco Interamericano de Desarrollo. 
KfW Development Bank \& InsuResilience Global Partnership. (2019). The Natural Disaster Fund (NDF) Deutschland was launched at the COP25 - InsuResilience. Retrieved from https://www. insuresilience.org/the-natural-disaster-fund-ndf-deutschland-was-launched-at-the-cop25/

Lemke, S. (2019). Soluciones Paramétricas Desafiando los límites del seguro tradicional: nuestras soluciones para Latinoamérica, el Caribe e Iberia. Retrieved from https://www.munichre.com/ content/dam/munichre/global/content-pieces/documents/MunichRe-Soluciones-ParametricasLatinoamerica-Caribe-Iberia.pdf/ jer content/renditions/original./MunichRe-SolucionesParametricas-Latinoamerica-Caribe-Iberia.pdf

Marulanda, M.C., Cardona, O.D., Mora, M. G. \& Barbat, A.H. (2014). Design and implementation of a voluntary collective earthquake insurance policy to cover low-income homeowners in a developing country. Natural Hazards, 74(3), 2071-2088. https://doi.org/10.1007/s11069-014-1291-4

McCord, M. \& Biese, K. (2015). El panorama de los microseguros en América Latina y el Caribe: Un mercado cambiante. Appleton, US: Microinsurance Network.

Miles, K.S. \& Wiedmaier-Pfister, M. (2018). Applying a Gender Lens to Climate Risk Finance and Insurance. Bonn: InsuResilience Global Partnership Secretariat.

Miles, K.S. \& Wiedmaier-Pfister, M. (2019). Integrating Gender Considerations into Different Models of Climate Risk Insurance (CRI). Bonn: InsuResilience Global Partnership Secretariat.

Miller, S. \& Keipi, K. (2006). Estrategias e instrumentos financieros para la gestión del riesgo de desastres en América Latina y el Caribe. Washington DC: Banco Interamericano de Desarrollo.

Ministerio de Hacienda y Crédito Público de Colombia. (2012). Estrategia financiera para disminuir la vulnerabilidad fiscal del estado ante la ocurrencia de un desastre natural. Paris: OECD. Retrieved from https://www.oecd.org/env/cc/CALDERON Estrategia Proteccion Financiera Desastres Naturales.pdf

Ministerio del Interior y Seguridad Pública de Perú. (2016). Ley 20920: Por la cual se aprueba la Política Nacional para la Gestión del Riesgo de Desastres. Lima: Ministerio del Interior y Seguridad Pública de Perú.

Narváez, L., Lavell, A. \& Pérez, G. (2009). La Gestión del Riesgo Un enfoque basado en procesos. Lima: Comunidad Andina. Retrieved from http://www.comunidadandina.org/predecan/doc/libros/ PROCESOS ok.pdf

National Academies of Sciences, Engineering, and Medicine. (2019). Attribution of Extreme Weather Events in the Context of Climate Change. Washington DC: NAP. https://doi.org/10.17226/21852

Raschky, P. \& Weck-Hannemann, H. (2007). Charity hazard-A real hazard to natural disaster insurance. Environmental Hazards, 7(4), 321-329. https://doi.org/10.1016/i.envhaz.2007.09.002

Schwarze, R. \& Wagner, G.G. (2004). In the Aftermath of Dresden: New Directions in German Flood Insurance. Geneva Papers on Risk and Insurance: Issues and Practice, 29(2), 154-168. https://doi. org/10.1111/i.1468-0440.2004.00279.x

Secretaría de Hacienda y Crédito Público de México. (2012). Estrategia Financiera Mexicana de Administración de Riesgos de Desastres. Tegucigalpa, Honduras: Secretaría de Hacienda y Crédito Público de México. Ciudad de México.

Swiss Re. (2020). Swiss Re: Our history. Retrieved from https://www.swissre.com/about-us/our-visionmission-and-values/our-history/established-1863.html

UNDRR. (2009). Terminología sobre Reducción del Riesgo de Desastres. Retrieved from https://www. unisdr.org/files/7817 UNISDRTerminologySpanish.pdf

UNGRD. (2017). Terminología sobre Gestión del Riesgo de Desastres y Fenómenos Amenazantes de Colombia. Retrieved from http://repositorio.gestiondelriesgo.gov.co/handle/20.500.11762/20761

Watson, C., Caravani, A., Mitchell, T., Kellett, J. \& Peters, K. (2015). Financiamiento para la reducción del riesgo de desastres: 10 cosas que debe saber. Londres: ODI \& UNDP. 